\title{
PCR survey of 50 introns in animals: Cross-amplification of homologous EPIC loci in eight non-bilaterian, protostome and deuterostome phyla
}

\author{
K. Gérard ${ }^{\mathrm{a}, 1}$, E. Guilloton ${ }^{\mathrm{b}, 1}$, S. Arnaud-Haond ${ }^{\mathrm{c}}$, D. Aurelle ${ }^{\mathrm{b}}$, R. Bastrop ${ }^{\mathrm{d}}$, P. Chevaldonné ${ }^{\mathrm{b}}$, \\ S. Derycke ${ }^{e}$, R. Hanel ${ }^{f}$, S. Lapègue ${ }^{g}$, C. Lejeusne ${ }^{h}$, S. Mousset', A. Ramšak, 'T. Remerie ${ }^{\mathrm{e}}$, F. Viard $^{\mathrm{k}}$, \\ J.-P. Féral ${ }^{b}$, A. Chenuil ${ }^{b, *}$
}

\begin{abstract}
a Laboratorio Ecología Molecular, las Palmeras 3425, Ñunoa, Santiago, Chile
b Institut Méditerranéen de Biodiversité et d'Ecologie marine et continentale, CNRS UMR 7263, Aix-Marseille Université, Station Marine d'Endoume, Chemin de la Batterie des Lions, 13007, Marseille, France

c Ifremer, Département Etude des Ecosystèmes Profonds-DEEP, Laboratoire Environnement Profond-LEP, Centre de Brest - BP 70, 29280 Plouzane, France

d University of Rostock, Institute of Biology, Albert-Einstein-Strasse 3, D-18059 Rostock, Germany

e Ghent University, Biology Department, Marine Biology Section, Krijgslaan 281 (S8), 9000 Ghent, Belgium

${ }^{f}$ Thünen-Institute of Fisheries Ecology, Palmaille 9, 22767 Hamburg, Germany

g Ifremer, SG2M-LGPMM, Laboratoire de Génétique et Pathologie des Mollusques Marins, Avenue de Mus de Loup, 17390 La Tremblade, France

${ }^{\mathrm{h}}$ Doñana Biological Station-CSIC, Wetland Ecology Departement, Avenida Américo Vespucio, s/n, 41092 Seville, Spain

'Université de Lyon, Université Lyon 1, CNRS, UMR 5558, 43 boulevard du 11 novembre 1918, F-69622 Villeurbanne, France

${ }^{j}$ National Institute of Biology, Marine Biological Station, Fornače 41, 6330 Piran, Slovenia

${ }^{k}$ UPMC Univ Paris 06, CNRS, UMR 7144 Adaptation \& Diversity in the Marine Environment Station Biologique de Roscoff, Place Georges Teissier, CS 90074, 29688 Roscoff, France

${ }^{1}$ Co-first authors

*: Corresponding author : Anne Chenuil, tel.: + 33491041617 ; fax: + 33491041635 ; email address : anne.chenuil@imbe.fr gerardkarin@yahoo.fr ; edith.guilloton@gmail.com ; Sophie.Arnaud@ifremer.fr ; didier.aurelle@imbe.fr ; ralf.bastrop@uni-rostock.de ; pierre.chevaldonne@imbe.fr ; s.derycke@UGent.be ; reinhold.hanel@ti.bund.de ; Sylvie.Lapegue@ifremer.fr ; lejeusne@ebd.csic.es ; mousset@biomserv.univ-lyon1.fr ; andreja.ramsak@nib.si ; thomas.remerie@gmail.com ; viard@sb-roscoff.fr ; jean-pierre.feral@imbe.fr ;
\end{abstract}

\begin{abstract}
:
Exon Primed Intron Crossing (EPIC) markers provide molecular tools that are susceptible to be variable within species while remaining amplifiable by PCR using potentially universal primers. In this study we tested the possibility of obtaining PCR products from 50 EPIC markers on 23 species belonging to seven different phyla (Porifera, Cnidaria, Arthropoda, Nematoda, Mollusca, Annelida, Echinodermata) using 70 new primer pairs. A previous study had identified and tested those loci in a dozen species, including another phylum, Urochordata (Chenuil et al., 2010). Results were contrasted among species. The best results were achieved with the oyster (Mollusca) where 28 loci provided amplicons susceptible to contain an intron according to their size. This was however not the case with the other mollusk Crepidula fornicata, which seems to have undergone a reduction in intron number or intron size. In the Porifera, 13 loci appeared susceptible to contain an intron, a surprisingly high
\end{abstract}


number for this phylum considering its phylogenetic distance with genomic data used to design the primers. For two cnidarian species, numerous loci (24) were obtained. Ecdysozoan phyla (arthropods and nematodes) proved less successful than others as expected considering reports of their rapid rate of genome evolution and the worst results were obtained for several arthropods. Some general patterns among phyla arose, and we discuss how the results of this EPIC survey may give new insights into genome evolution of the study species. This work confirms that this set of EPIC loci provides an easy-to-use toolbox to identify genetic markers potentially useful for population genetics, phylogeography or phylogenetic studies for a large panel of metazoan species. We then argue that obtaining diploid sequence genotypes for these loci became simple and affordable owing to NextGeneration Sequencing development. Species surveyed in this study belong to several genera (Acanthaster, Alvinocaris, Aplysina, Aurelia, Crepidula, Eunicella, Hediste, Hemimysis, Litoditis, Lophelia, Mesopodopsis, Mya, Ophiocten, Ophioderma, Ostrea, Pelagia, Platynereis, Rhizostoma, Rimicaris), two of them, belonging to the family Vesicomydae and Eunicidae, could not be determined at the genus level.

Keywords: Universal primers ; Alternative barcoding ; Non-model species ; Genetic marker ; Intron 


\section{Introduction}

Population genetics and genomics of non-model species (including ecologically relevant model species) are hampered by the lack of knowledge of their genome and the absence of universal primers (e.g. Chenuil 2006). This is a particular problem for phyla of marine invertebrates which encompass a much wider phylogenetic range than terrestrial metazoans. Next-Generation Sequencing (and, to a lesser extent, Next-Generation Genotyping) methods underwent a significant diversification and decrease in cost. With respect to population genetics, the starting material may be mRNA, good quality genomic DNA for Rad-Seq (Narum et al 2013), or PCR products (amplicons). Amplicons remain the most convenient solution relative to field sampling constraints; they also still correspond to the cheapest approaches when hundreds of markers are not requested. In particular, with the development of biodiversity studies using barcoding and metabarcoding and the need of multilocus data, the need of universal primers for rarely studied phyla is growing. Introns are non-coding genomic regions susceptible to provide highly variable molecular markers. Primer pairs were recently designed to amplify introns in a very wide phylogenetic spectrum of species; the design was based on the choice of intron positions that are well conserved across metazoan phyla and which were embedded within highly conserved exon sequences which do not appear duplicated in annotated genomes (Chenuil et al., 2010). About 50 introns, framed by one or several alternative primer pairs in exons, were tested for PCR amplification and an average of 24 introns per species appeared promising in Bilaterian species. Among those promising introns, five were amplified successfully in all 10 species including cnidarians. Some of these loci were sequenced in numerous individuals and proved useful for population genetic and phylogeographic studies (Penant et al., 2013; Pivotto et al. in prep.). By providing nuclear markers in non-model species, these loci allowed for example disentangling intricate phylogeographic situations within species complexes like the sea urchin Echinocardium sp. (Egea, 2011; Egea et al., unpublished), the gastropod Hexaplex trunculus (Marzouk et al., unpublished) and the cockle Cerastoderma glaucum (Chenuil \& Tarnowska, unpublished). They also provided codominant nuclear markers such as microsatellites useful for populations genetic studies in different species, e.g. the sea urchin Abatus cordatus (Ledoux et al., 2012), or the brittlestar Ophioderma longicauda (Weber et al., submitted). In the present study, we aimed to extent this EPIC survey to additional phyla. We designed more than 70 additional alternative primers for the same set of loci and we investigated their amplification patterns in 23 species, not tested previously, from seven different phyla. The phyla were chosen to encompass a very wide phylogenetic spectrum. They included the two main non-bilaterian phyla, Porifera and 
81 Cnidaria, and the most diverse bilaterian phyla. We surveyed four Protostomian phyla (i.e. two

82 Ecdysozoan phyla, Arthropoda and Nematoda, and two Lophotrochozoan phyla, Mollusca and Annelida) and a Deuterostomian phylum (Echinodermata). Another Deuterostomian phylum, Urochordata, had been investigated in a previous study (Chenuil et al 2010), and for Vertebrata, numerous markers including EPICs (Atarhouch et al 2003) are already available.

\section{Materials and methods}

The method for primer design and the sequences of previously designed primers were given in Chenuil et al. (2010). New primers were specifically designed in this study in order to improve complementarity with ecdysozoan (i.e. arthropods and nematodes) and cnidarian genomes (but not for poriferans (i. e. sponges). For those phyla, new expressed sequenced tags (EST) sequences were aligned with genome sequences of the gene families previously selected for EPIC design by Chenuil et al. (2010). The set of new primer sequences (several combinations were tested) is given in Table 1. The alternative primers we designed (for a given locus and a given amplification direction) most often corresponded to different levels of ambiguity for the same positions, and in some cases to a slight positional shift (Table 1). The PCR reaction contained the following quantities: 2. $4 \mu \mathrm{l}$ sterile distilled water, $2.5 \mu \mathrm{l}$ of $\mathrm{MgCl} 2$ at $25 \mathrm{mM}, 2.5 \mu \mathrm{l}$ of $5 \mathrm{X}$ green buffer (flexi-go taq Promega), $2 \mu \mathrm{l}$ of a mixture of dNTP ( $0.2 \mathrm{mM}$ each), $0.25 \mu \mathrm{l}$ of a solution of $50 \mu \mathrm{M}$ for each primers, $0.06 \mu \mathrm{l}$ of flexi GoTaq ${ }^{\circledR}$ polymerase (concentrated at $5 \mathrm{u} . \mu \mathrm{L}-1$ ) and $1 \mu \mathrm{l}$ of DNA extract at $5 \mathrm{ng} . \mu \mathrm{l}^{-1}$. The PCR program was: $2 \mathrm{~min}$ at $94^{\circ} \mathrm{C}$; 14 cycles of $1 \mathrm{~min}$ at $94^{\circ} \mathrm{C}, 1 \mathrm{~min}$ at hybridization temperature from $58^{\circ} \mathrm{C}$ for the first cycle to $45^{\circ} \mathrm{C}$ for the $14^{\text {th }}$ cycle, 1 min at $73^{\circ} \mathrm{C}$; 25 cycles of $40 \mathrm{sec}$ at $94^{\circ} \mathrm{C}, 40 \mathrm{sec}$ at $58^{\circ} \mathrm{C}, 1 \mathrm{~min}$ at $72^{\circ} \mathrm{C}$, and finally $3 \mathrm{~min}$ at $73^{\circ} \mathrm{C}$. For each sample, $5 \mu \mathrm{l}$ of PCR products were checked on large 1.5\% agarose gel electrophoresis as in Chenuil et al. (2010). For small sized species, i.e. Hemimysis margalefi and Litoditis marina, DNA extracts from distinct specimens had to be used for different sets of EPIC loci. DNA extraction methods varied according to organisms: DNeasy tissue kits (Qiagen) were used for all cnidarian and ophiuroids species, QiaAMp DNA minikit (Qiagen) for Acanthaster, Aplysina, Hemimysis and Platynereis, Nucleospin ${ }^{\circledR}$ Multi-96 Tissue Kit (MACHEREY-NAGEL) were used for Crepidula fornicata, CTAB protocols with proteinase-K incubation at $55^{\circ} \mathrm{C}$ were used for the Vesicomydae sp., the Eunicidae sp., Rimicaris and Alvinocaris spp. (Doyle \& Doyle 1990, Teixera et al, 2013), a customized CTAB protocol (Remerie et al 2006) for Mesopodopsis, a protocol explained in Derycke et al (2005) for Litoditis (which was named Pellioditis) and the innuprep DNA minikit (Analytik Jena) for Hediste. After excluding individuals that were not amplified for any intron, the result for each primer pair in each species was 
classified into one of three categories: (1) P (promising) which corresponds to amplification in all individuals of the species, without multiple bands, and of sufficient size to potentially contain an intron of at least $70 \mathrm{bp}$ (the expected size of a putative intron after removal of the exonic fragment are reported for each primer pair in Chenuil et al. (2010)); (2) I (intron) corresponds to less intense amplifications or cases with multiple bands; (3) A (amplification) correspond to other cases resulting in amplification products, yet particularly amplicons which are too small to contain an intron, and excluding those producing only primer dimers or small size artefactual amplification products. However, we cannot exclude that occasionally some particularly large artefactual amplification products were erroneously classified as "A" results, since we did not sequence the amplicons. A precise estimation of the frequency of such mis-classifications is not available, but amplicons from about two dozens of different loci or species (including two 'A' loci) were sequenced by some of us and other colleagues and the results always provided sequences embedded within the expected exonic sequence (unpublished or cited in introduction). In one or two cases, we also observed, among the sequenced clones, an artefactual sequence not embedded in the expected exonic regions (unpublished data) which was smaller. DNA extracts from different species were distributed among three different 96-well plates, for which we did not test exactly the same combination of primer pairs for each locus. Each combination of forward and reverse primer was given a name reported in Table 2. Primer pairs tested for each plate appear in Table S1 (Supplementary material). The plate "ECDY-Platy” was mainly composed of samples from ecdysozoans, and for this plate we preferentially tested the new primers specially designed for ecdysozoans (a total of 69 primer pairs was tested). For logistic reasons (i.e. filling of 96-well plates, to allow the use of multichannel pipets and to limit the number of agarose gels), we also used two non-ecdysozoan DNA samples in this plate, corresponding to Platynereis dumerilii (Polychaeta) which were thus tested using the same primer pairs, a priori nonoptimal for this taxon. The plate "CNI-POR-Hedi” contained a majority of cnidarians but also two non-cnidarian species, Hediste diversicolor (Polychaeta) and Aplysina cavernicola (Porifera). Some primers designed for cnidarians were preferentially used for this plate, which was tested with 68 primer pairs. The third plate contained exclusively lophotrochozoans (mollusks and polychaetes) and echinoderms (named “LOPHO-ECHI”) and was used for 75 primer pairs. The number of samples for each species is given in parenthesis after the species name. In the plate « ECDY-Platy », we tested the nematode Litoditis marina (2), the arthropods Rimicaris exoculata (4), Alvinocaris muricola (3) and Alvinocaris markensis (3) which afterwards appeared to belong to the same species (Teixeira et al., in press), Hemimysis margalefi (3), Mesopodopsis slabberi (4), and the polychaete Platynereis dumerilii (2). In the plate “CNI-POR-Hedi”, we tested the cnidarians Eunicella cavolinii (3), Eunicella verrucosa (2), Lophelia pertusa (4), Pelagia noctiluca (2), Rhizostoma pulmo (2), Aurelia 
aurita (2), but also Aplysina cavernicola (Porifera) (1) and Hediste diversicolor (Polychaeta) (4). In the "LOPHO-ECHI” plate, we tested the echinoderms Acanthaster planci (2), Ophiocten sericeum (3), Ophioderma longicauda (3), the mollusks Crepidula fornicata (4), Vesicomyidae sp. (1), Ostrea edulis (3), and the polychaetes Eunicidae spp. (3), and Platynereis dumerilii (2) for which some samples were also tested in the plate "ECDY-Platy", i.e. with slightly different primer pairs for some loci. After these tests, a new plate (named "IV (i21-i51)”) has been composed of a variety of samples from the former plates for which we increased or decreased the DNA amount (3-fold increase for Hemimisys and Mesopodopsis, 3-fold dilution for Crepidula), and from an additional mollusk species, Mya arenaria (4 specimens), and to be tested exclusively with two loci (i21 and i50) that appeared particularly successful in (Chenuil et al., 2010) with the original set of primers, excluding newly designed primers (supposedly adapted to ecdysozoan or cnidarian). The other ecdysozoan species were also tested in this plate, without changing their DNA concentrations (Litoditis, Rimicaris and the two Alvinocaris species).

\section{Results}

The results for each species across the set of loci vary considerably among species (see Table 3 for detailed results and Table 4 for a summary per species). The best results were obtained for the oyster with 28 loci providing amplicons of sufficient size to contain an intron. The sponge $A$. cavernicola successfully amplified 13 distinct EPIC loci with amplicon sizes suggesting the presence of an intron ( $\mathrm{P}+\mathrm{I}$ results). This is noteworthy as no sponge genome sequence data were used when we designed primer sequences (Chenuil et al., 2010). The Porifera phylum branches before all the other phyla surveyed in the tree of life and phylogenetic divergence is a major parameter influencing primer design efficiency. In the two cnidarians of the genus Eunicella, we obtained 24 loci with intron size amplicons, despite their phylogenetic distance with genomes that most influenced primer design.

Three Ecdysozoans globally did not provide good and regular amplification, in particular Hemisysis and Mesopodopsis, yet the two deep sea shrimps Alvinocaris and Rimicaris obtained good results. In plate "i21-i51” for which we used the initial set of primers from (Chenuil et al., 2010) instead of the newly designed primers based on ecdysozoan ESTs, we obtained better results in some cases (i.e. in Litoditis and Rimicaris, for which DNA concentration were unchanged) but not always. 
The gastropod Crepidula fornicata displayed a significantly higher proportion of amplicons too short to contain an intron as compared to the average computed across the other species (exact test, $\mathrm{p}<0.001$ ) (Table 4). This proportion is even more extreme in the arthropod Hemimysis and is also high in the arthropod Mesopodopsis and the nematode Litoditis but since few primer pairs amplified in this species (4 to 8), the estimated proportion of short amplicons is not precise at all.

\section{Discussion}

The good results obtained for cnidarians are not due to the design of special primers using cnidarian EST information, because, contrary to the ecdysozoans for which most primers were newly designed, few newly-designed primers were used for cnidarians (Table 1 -Table S1). This, together with the good results obtained for the sponge, confirms that our approach enables finding candidate loci across the genome, for species for which only very few polymorphic markers are available, across a very wide phylogenetic range.

Attempts to reconstruct phylogenetic trees (not shown) based on the amplification patterns ("P”, "I” and “A” contingency tables) obtained for each locus evidenced a strong influence of the DNA plate (thus of the primer pair combinations), and of the proportion of successful loci per species, species with good results being grouped together (and the reverse). Within genera (i.e. the two Eunicella species, and the Alvinocaris species) the results were highly similar though not strictly identical (Table 2). At a higher taxonomic level however, we found no influence of taxonomical relatedness. As a consequence, to identify, for a new species, potentially useful EPIC loci from our set of markers, it is recommended to first test the primer pairs that globally appeared as the best one on the whole range of phyla tested, rather than to choose those that worked in the most closely related taxa (except if congeneric species or close genera were surveyed). Those "first choice” loci appear on Table 3 (e.g. locus i50) and generally correspond to the best ones identified in Chenuil et al. (2010).

The contrasted patterns observed across the study taxa may be explained by several possible causes (Table 5). Firstly, DNA damage is expected to decrease the number of successfully amplified loci (leading to low values of the triplet $(\mathrm{A}+\mathrm{P}+\mathrm{I})$ ), and increase the proportion of short amplicons (A) among successful amplifications, because short fragments are more likely to remain intact in target DNA. Secondly, high evolutionary rates are expected to increase mispriming of the PCR primers, decreasing the number of successfully amplified loci. This process would equally affect the loci containing an intron or not and consequently the proportion of short amplicons should not be 
211 influenced. Finally, natural selection for a reduction of intron length in a genome should turn patterns 'P' and 'I' into 'A', but should not decrease the amount of successful loci. Those three hypotheses lead to different patterns and can theoretically be distinguished (Table 5). Natural selection favouring large introns, contrary to selection for small introns, seems unlikely to affect the genome globally and is a less relevant hypothesis to explain the proportion of P, I and A results of a taxon; however in case it occurs, this would significantly decrease the amount of amplifying loci, since we rarely obtained amplicons exceeding 1000 bp with our experimental conditions (this corresponds to intron sizes between 720 and 930 bp, most often of 850 bp after removing the exonic fragment length). Comparing the results obtained for the different taxa (Table 4) with the three scenarios above (Table 5), we suggest that Crepidula introns may have been affected by natural selection for length reduction. One hypothesis that has been proposed to explain introns evolution is linked to life cycle parameters such as generation duration (Jeffares et al., 2006). Crepidula fornicata is a perennial species -living 8-12 years- but some authors have hypothesized that it may be better described as a species with an r-strategy life cycle (Richard et al., 2006). In such species intron loss may allow replication time reduction (Jeffares et al., 2006). Although large-scale ESTs libraries were built-up (Henry et al. 2010) for this species, there is no genome data available for this species to confirm this scenario. The patterns displayed by two arthropods (Hemimysis and Mesopodopsis) and the nematode Litoditis at first sight are best explained by damaged DNA, and these species are the smallest of the survey. Note however that in the case of Litoditis the second scenario cannot fully be rejected as this species is a very strong colonizer (r-strategy) and this may contribute to its high proportion of short amplifications due to reduction in intron size. In the "i21-i51" plate, DNA concentration was doubled for these two arthropods resulting in a gain of amplification, for locus 21, for one of those two species, whereas i50 remained unamplified; therefore the influence of DNA quantity for these samples is not clearly established. However, an influence of DNA quality on our results is strongly supported by the profiles of DNA extracts on agarose gels: the oyster samples displayed, by far, the best profiles (a very neat band of high molecular weight and no degradation smear), and Hemimysis and Mesopodopsis displayed degraded migration profiles (though comparable to those from other species that performed better on PCR tests). Nematodes and Arthropods generally display less and smaller introns and appear to have lost them (Cho et al., 2004; Hawkins, 1988; Rogozin et al., 2003). By contrast with ecdysozoans, Platynereis (Raible et al., 2005) and the cnidarians (Zimek and Weber, 2008) were reported to have highly conserved genome sequences and intron-exon structures (our own experience based on their nucleotide alignments supports this view). However, three of the arthropods we surveyed displayed numerous successfully amplified loci and a low proportion of amplicons too short to contain an intron, as the majority of the species. While we designed new 
primers, we observed a lot of variation among arthropods, more than within other phyla and we actually expected that those new primers may not improve PCR efficiency. The annelid Platynereis which was tested both in the ECDY-Platy plate (with numerous ecdysozoan primers) and in the LOPHO-ECHI plate (mostly with the initial set of primers), obtained slightly more amplification results (P, I or A) in the ECDY-Platy plate (22 versus 19), suggesting that the bad results obtained for some ecdysozoan species are not directly explained by the design of the new PCR primers dedicated to ecdysozoans, but rather by their high evolutionary rate or a global intron reduction (Raible et al 2005, Zimek and Weber 2008).

This second survey of the EPIC loci isolated in Chenuil et al (2010) confirms that those EPIC primers may potentially amplify any metazoan species. Combining the present and the former study, some loci appear more likely to successfully amplify an intron : i1, i2, i5, i8, i9, i11, i21, i34, i36, i50 for Mollusks (five species, four genera tested), i5, i12, i15, i22, i29, i30, i53 for Cnidarians (eight species, seven genera), i1, i2, i5, i9, i21, i22, i29, i36, i50, i51 in echinoderms (eight genera), and i26, i29, i35, i50, i51 for Arthropods (four genera). We emphasize however that amplification results are very poorly correlated to phylogeny and it is strongly recommend to test all the EPIC loci (if possible, combining several species for the same session).

Recently, Li et al. (2013) developed a hybridization capture method which allows finding hundreds of coding sequences in highly divergent vertebrate species. This promising method however does not target highly variable genomic regions. Furthermore, it is more complex and expensive than an EPIC PCR survey, even when PCRs are followed by a Next-Generation Sequencing run. For example, amplicons from all intronic loci can be pooled in a MISEQ run using up to 184 tags to label the different individuals. For about $3000 €$ one can obtain more than ten millions of paired-end reads (250 bp x 2 each) for 96 tagged individuals, resulting in more than 1000 paired-end reads per locus for each individual in average. With such a sequencing depth, diploid sequence genotypes can be safely inferred as explained in Chenuil (2012): in particular, the analysis of the distribution of read numbers within individuals allows detecting whether a marker corresponds to a single and diploid locus or whether there is polyploidy or paralogy, and allows determining the level of multiplication; loci prone to (and alleles generated by) PCR or sequencing errors also are identifiable using such distributions. 
275

276

277

278

279

280

281

282

283

284

285

\section{Acknowledgements}

The authors acknowledge the support of the consortium GeBiRM, within the Network of Excellence 'Marine Biodiversity and Ecosystem Functioning' (MarBEF) which was funded by the Sustainable Development, Global Change and Ecosystems Programme of the European Community's Sixth Framework Programme (contract no. GOCE-CT-2003-505446). Maciej Wolowicz is thanked for providing DNA extracts of Mya. Additional support for the molecular work was obtained from the network Marine Genomics Europe (GOCE-CT-2004-505403).

\section{References}

Atarhouch, T., Rami, M., Cattaneo-Berrebi, G., Ibanez, C., Augros, S., Boissin, E., Dakkak, A., Berrebi, P., 2003. Primers for EPIC amplification of intron sequences for fish and other vertebrate population genetic studies. Biotechniques 35, 676-682.

Chenuil, A., 2006. Choosing the right Molecular Genetic Markers for studying biodiversity: from molecular evolution to practical aspects. Genetica 127,101-120.

Chenuil, A., 2012. How to infer reliable diploid genotypes from NGS or traditional sequence data: from basic probability to experimental optimization. Journal of Evolutionary Biology 25,949-960.

Chenuil, A., Hoareau, T.B., Egea, E., Penant, G., Rocher, C., Aurelle, D., Mokhtar-Jamai, K., Bishop, J.D.D., Boissin, E., Diaz, A., Krakau, M., Luttikhuizen, P.C., Patti, F.P., Blavet, N., Mousset, S., 2010. An efficient method to find potentially universal population genetic markers, applied to metazoans. Bmc Evolutionary Biology 10.

Cho, S., Jin, S.W., Cohen, A., Ellis, R.E., 2004. Aphylogeny of Caenorhabditis reveals frequent loss of introns during nematode evolution. Genome Research 14, 1207-1220.

Derycke, S., Remerie, T., Vierstraete, A., Backeljau, T., Vanfleteren, J., Vincx, M., Moens, T., 2005. Mitochondrial DNA variation and cryptic speciation within the free-living marine nematode Pellioditis marina. Marine Ecology Progress Series 300, 91-103.

Doyle, J.J., Doyle, J.,L., 1990. Isolation of plant DNA from fresh tissue. Focus 12, 13-15. 
Egea, E. 2011. Histoire évolutive, structures génétique, morphologique et écologique comparées dans un complexe d'espèces jumelles: Echinocardium cordatum (Echinoidea, Irregularia). PhD Thesis. Université de la Méditerranée, Aix-Marseille.

Hawkins, J.D., 1988. A survey on intron and exon lengths. Nucleic Acids Research 12, 9893-9908.

Henry, J. J., R. Collin, and K. J. Perry. 2010. The slipper snail, Crepidula: an emerging lophotrochozoan model system. Biological Bulletin 218:211-229.

Jeffares, D.C., Mourier, T., Penny, D., 2006. The biology of intron gain and loss. Trends in Genetics 22, 1622.

Ledoux, J.B., Tarnowska, K., Gerard, K., Lhuillier, E., Jacquemin, B., Weydmann, A., Feral, J.P., Chenuil, A., 2012. Fine-scale spatial genetic structure in the brooding sea urchin Abatus cordatus suggests vulnerability of the Southern Ocean marine invertebrates facing global change. Polar Biology 35, 611623.

Li, C., Hofreiter, M., Straube, N., Corrigan, S. and Naylor, G.J.P., 2013. Capturing protein-coding genes across highly divergent species. BioTechniques 54, 321-326.

Narum, S. R., Buerkle, C. A., Davey, J. W., Miller, M. R. and Hohenlohe, P. A., 2013. Genotyping-bysequencing in ecological and conservation genomics. Molecular Ecology 22: 2841-2847.

Penant, G., Aurelle, D., Féral, J.-P., Chenuil, A., 2013. Planctonic larvae do not ensure gene flow in the edible sea urchin Paracentrotus lividus. Marine Ecology-Progress Series 480:155-170.

Raible, F., Tessmar-Raible, K., Osoegawa, K., Wincker, P., Jubin, C., Balavoine, G., Ferrier, D., Benes, V., de Jong, P., Weissenbach, J., Bork, P., Arendt, D., 2005. Vertebrate-Type Intron-Rich Genes in the Marine Annelid Platynereis dumerilii. Science 310, 1325-1326.

Remerie, T., Bourgois, T., Peelaers, D., Vierstraete, A., Vanfleteren, J., Vanreusel, A., 2006. Phylogeographic patterns of the mysid Mesopodopsis slabberi (Crustacea, Mysida) in Western Europe: evidence for high molecular diversity and cryptic speciation. Marine Biology 149, 465-481.

Rogozin, I.B., Wolf, Y.I., Sorokin, A.V., Mirkin, B.G., Koonin, E.V., 2003. Remarkable interkingdom conservation of intron positions and massive, lineage-specific intron loss and gain in eukaryotic evolution. Current Biology 13, 1512-1517.

Teixeira, S., Olu, K., Decker, C., Cunha, R. L., Fuchs, S., Hourdez, S., Serrão, E.A. and Arnaud-Haond, S., 2013. High connectivity across the fragmented chemosynthetic ecosystems of the deep Atlantic Equatorial Belt: active dispersal or dubious endemism? Molecular Ecology (doi: 10.1111/mec.12419) 
Raible, F., Tessmar-Raible, K., Osoegawa, K., Wincker, P., Jubin, C., Balavoine, G., Ferrier, D., Benes, V., de Jong, P., Weissenbach, J., Bork, P., Arendt, D., 2005: Vertebrate-type intron rich genes in the marine annelid Platynereis dumerilii. Science 310,1325-1326.

Zimek, A., Weber, K., 2008. In contrast to the nematode and fruit fly all 9 intron positions of the sea anemone lamin gene are conserved in human lamin genes. European Journal of Cell Biology 87, 305-309.

\section{$\underline{\text { Mini-CVs of authors }}$}

- KG is an evolutionary biologist interested in biogeographic processes that drive SouthernHemisphere marine diversity especially in Antarctic waters.

- EG is a research assistant on eel conservation. She is interested in evolution and conservation, particularly in catadromous fish.

- SAH is an evolutionary ecologist with a strong interest in the understanding of factors involved in the divergence of populations, particularly in the marine environment.

- DA is an evolutionary biologist interest in population genetics and adaptive processes

- $\quad$ RB is a senior scientist at the university of Rostock, working on phylogeny and phylogeography, biological invasions, ecophysiology.

- PC is a Senior CNRS researcher working primarily on the Mediterranean marine biodiversity, with an emphasis on underwater cave biota and their relation with deep-sea communities. Among model organisms studied are sponges, cnidarians, echinoderms and mysid crustaceans.

- Sofie Derycke is a postdoctoral fellow. Her research focus is on population genetics, phylogeography, cryptic speciation and taxonomy of marine invertebrates. She also has a keen interest in assessing taxon and functional diversity of marine sediments using DNA barcoding, metagenetics and metagenomics and in linking this diversity to ecosystem stability and resilience.

- $\quad$ RH is head of the German Federal Research Institute of Fisheries Ecology in Hamburg. He is a marine biologist interested in causes and pathways of adaptation and speciation in the sea. 
- SL is geneticist and is developing research in population genetics/genomics and selective breeding of marine molluscs.

- CL is a molecular ecologist studying the effects of large scale disturbances (mainly climate change and biological invasions) on biodiversity and the adaptive responses of organisms.

- $\mathrm{SM}$ is a population geneticist. He is interested in population genomics, forces shaping genetic variation in populations and species and in methods related to the analysis of sequence polymorphism.

- AR Andreja Ramšak is a molecular biologist and her research interest is focused on phylogeography and population genetics of scyphozoans.

- $\quad$ TR finished his PhD in Marine Biology at Ghent University, and participated in several research projects on marine genetic biodiversity (phylogeography, population genetics, phylogeny of marine invertebrates). In 2009 he started as a lecturer at the Artevelde University College, where he is involved in natural sciences education, as part of the teacher training program.

- F.V. is a senior CNRS researcher. Her interests include molecular ecology and evolutionary biology to examine dispersal and adaptation processes of marine coastal species, in particular invasive species.

- J-P.F is a senior CNRS researcher. He aims to understand the origin, the maintenance and the erosion of biodiversity taking into account the mode of development of marine benthic invertebrates and environmental factors in continuous and insular systems.

- AC is an evolutionary biologist working on population genetics, population genomics and phylogeography of marine organisms. 
Table 1: New primers (altogether 104) designed for this study. Nucleotides at ambiguous sites are marked using the IUPAC ambiguity code.

\begin{tabular}{|c|c|}
\hline i1F2 & GAATCAGGCCTGTCCATGGTNAVBTGG \\
\hline i1R3 & TGGCCATATTCCATTGACCAAATGMAYTTRAAYTC \\
\hline i3F2 & TTGATTTGGCGTATGCTATCGAACARATGTGGSA \\
\hline i3R2 & CAACTGTCAGCAATTACTAACAKYTCRTKRTA \\
\hline i4F2 & ATCTAGAGCTCATCATAGATTTACAGGRSCNCARAT \\
\hline i4R2 & GTTTTCGGTCTTAATATTCATAARRTTCATNCC \\
\hline i5F2 & TGTTCCCAGCAGAATATCCNATGMARCC \\
\hline i5R3 & CATATTTTCTTGTTTAATTCAAAACGHCCATTHGG \\
\hline i5R4 & TCCATGATGGTTGCCATGTTTCYGGRTGRTR \\
\hline i5R5 & TCCATGATGGTTGCCATGTYTCYGGRTGRT \\
\hline i8F2 & TTCCAGTGGTCATGTGGCATGGMATGGGYGA \\
\hline i8R2 & CTATTTTTCCCAAACTTAATGGRTTRCARCA \\
\hline i9F3 & TGCCTCTCCATTTCCGGCTATCAYCCRGARAC \\
\hline i9R2 & TATAGCGCCCTCTCCTTTGGTAGGCAKRAANSCAAT \\
\hline i11F2 & TATGTTTTTGGTTGGAATGCAGRAYAARAARAT \\
\hline i11R2 & ACTGCCTGCAAGTGACGATCRTAYTCYTG \\
\hline i12F2 & GATGATAAAAGTGTCAGARTNTGGGARTGGGA \\
\hline i12R2 & TGAAGTCCAACATTTTGAATAAGTTTYRTRTCNAC \\
\hline i13F2 & TGGGTGCTCATTGGACACGARTWYATGGA \\
\hline i13R2 & ATAATATCATACATTTGTCCAARNCCRTACCA \\
\hline i17F2 & ATTGGTGTATATATTATAGAYMGDTAYAC \\
\hline i17R2 & ATGTTGGAAGATTCGCGAAGATCCRAARAARTC \\
\hline i19F2 & GAAACCGATTGATGTGAAAACAAARTTYTAYARYGC \\
\hline i19F3 & GAAACCGATTGATGTGAAAACAAAGTTYTAYARYGC \\
\hline i19R3 & TGTATTGTTCCGAACTTTCAAGTTCSACCTTYTCSAG \\
\hline i21F3 & AAAACCAATTTACAATCCTGCTGGAAARTAYAYGWT \\
\hline i21R3 & GATCCAGGAAAGTCATATCCTCCCATAASYTTCATRTA \\
\hline i21R4 & GATCCAGGAAAGTCATATCCTCCCATNSTTCATRTA \\
\hline $\mathrm{i} 22 \mathrm{~F} 3$ & GCTGCTGGAGAAGGCCTACATKAARGTSAT \\
\hline i22F2 & TACATGAAGGTTATGGGAGGVTAYGAYTT \\
\hline i22R2 & GTATCGTTCAATTCAATTCTTTCHGGWATCCA \\
\hline i22R3 & CAATTCAATTCTTTCAGGAATCCADCCBGTYA \\
\hline i24F2 & AAGAGTTTATCACTCTTATTGTGTAYRAVAVY \\
\hline i24R3 & GAATAGTTGATTGTATTGGTTTTYTCRTAYTG \\
\hline i25F3 & AGCGTGGATGGACACCTGAARTTYTGGAARAA \\
\hline i25R3 & TCCAGCTTTATCATGTTGATCATRTCRAARTT \\
\hline i26F3 & TGATGAATGTCCGAAAACCGTKGARAAYTTCTG \\
\hline i26R3 & AAATTCATCTTCAAAATCTCYDCCCCADAT \\
\hline
\end{tabular}


Table 1 (continued)

\begin{tabular}{|c|c|}
\hline i29F2 & ATCGGTGATCGATTTCGATGAGATSGCGGHGG \\
\hline i29F3 & GATTTCGATGAGATGGCCGGAGGTBYVAACAA \\
\hline i29R2 & GTTTTACCGGATCCCTGCAAACCNACRAACATKA \\
\hline $\mathrm{i} 29 \mathrm{~F} 4$ & ATGGCAGCTGGTCTCAACAAACGCARRATGATHCAR \\
\hline i30F2 & TTCCGTGCTGGTGCTTTCGATCAAATMAARCARAAYGC \\
\hline i30F3 & TTCCGTGCTGGTGCTTTCGATCAAATAAARCARAAYGC \\
\hline i30R2 & GTATCCACAATGATGATTTCRAANCCYTC \\
\hline 30R3 & CCGCTAGTATCCACAATGATGAHYTCRAARTT \\
\hline i34F2 & GACATGTATGAGCAGTTCCAGAACATYATGAARATGGG \\
\hline i34R3 & TTCCTTATCACTCATAGTGTCCATSAYNGTCAT \\
\hline 34R4 & TTCTTCATCCTTCATACTGTCCATBATNRTCAT \\
\hline i35F3 & CAATACAAGAAATTCTCTGCTGTGGTAAAGAARATKGG \\
\hline i35F4 & CAATACAAGAAATTCTCTGCTGTGGTHAAGAARATKGG \\
\hline i35R3 & GGATCCATCATTTTTGCCATYTGHTGRTT \\
\hline i35R4 & GGATCCATCATTTTTGCYATYTGHTGRTT \\
\hline i36F2 & TTCAAGGGAACCATCATGGAAGARTGGTWYTTY \\
\hline i36R3 & ACGTTTCCGCTGAGTACATTTGCTGGSAWCATYTG \\
\hline i37F3 & TGTCGAACATTCTTCTCCACNCAYTAYCA \\
\hline i37F2 & TGTCGAACATTCTTCTCCACNCAYTATCA \\
\hline i37R2 & GGATCCTCATTGTTCTCCTTATCCACCATGCANKY \\
\hline i38F2 & AACGCGAGAGTTCGTGTTCTCACVTACACYGAYGA \\
\hline i38R2 & GATCCGGATGGTTATTGAACCAYACKCCRTACATR \\
\hline $\mathrm{i} 41 \mathrm{~F} 2$ & AACCCAATGGAGGCCTATTACTTCACDGTRGC \\
\hline $\mathrm{i} 41 \mathrm{~F} 3$ & CGTGGAACCCAATGGAGGCCTATTACTTCACDGTR \\
\hline i41R3 & ACTGGATGCTCCATATAACGCATGTCRWABGTRTA \\
\hline i41R4 & GACGGAGGCCATTCGTTTTGTGTAGTADAYDTCYC \\
\hline $\mathrm{i} 42 \mathrm{~F} 2$ & GGAAAACGATTGGTAATGTTYGGMAARTR \\
\hline i42R3 & GCCAATCCCATGTGAAGGAAYGGKGTRTKRTG \\
\hline i42R4 & TTAGCTGCACGACTGCTCTTGTARTTRTGNG \\
\hline i43R3 & CAATATGGGTTTCGACCGTGATGKACMCKRTGATG \\
\hline $\mathrm{i} 44 \mathrm{~F} 2$ & AGAATAAAATTTATAGATCTTATATYGGAATGGGW \\
\hline i44F3 & AGAATAAAATTTATAGATCTTAYATYGGAATGGGW \\
\hline i44R2 & CCCTGTGAGATTTCTGCTTGGTATGGDTRTACTG \\
\hline i44R3 & CCCTGTGAGATTTCTGCTTGGTAHGGDGTRTACTG \\
\hline 44F4 & AGAATAAAATTTATAGATCTTATATYGGMATGGGNTA \\
\hline i45F2 & CAAGTTTATTTGGATGGAGCCAAYATGAATGCYC \\
\hline i45F3 & CAAGTTTATTTGGATGGAGCYAAYATGAATGCYC \\
\hline i45R2 & GGACCACCTCCTCCGTGTGGAATRCARAAKGT \\
\hline i45R3 & GGACCACCTCCTCCGTGTGGWATRCARAAKGT \\
\hline $\mathrm{i} 46 \mathrm{~F} 2$ & CGAAGTACACAAATTCCGTTGGGARACNTGYTG \\
\hline i46R2 & GCCGCATTATTCTTCATTTCCATRAAYTCRTG \\
\hline i47F2 & GACAGTGAGCATGCGATCAAGTTCTTYCARMGVGC \\
\hline i47R2 & ATGATATCATACATTTGGCCGARNCCRTACCA \\
\hline
\end{tabular}


Table 1 (end)

$\begin{array}{ll}\text { 48F4 } & \text { GGAGATTATGAAAATGCTGAGAAGMWHTGYATGCW } \\ \text { i48F2 } & \text { CAATCAGGAAATTATGTGGAAGCAGAAARRYWTG } \\ \text { i48F3 } & \text { CAATCAGGAAATTATGTGGAAGCAGARARRYWTTG } \\ \text { i48R2 } & \text { GCTGCGGCTAAATTGATGTAACCATCAATRAAWTC } \\ \text { i49F3 } & \text { GGAAAACTAAACGACGCCATACTCCAYTAYAARGA } \\ \text { i49R3 } & \text { ATTCGAATGAGCATCGGCAAATGCTGGRTTRATYT } \\ \text { i49R4 } & \text { ATTCGAATGAGCATCGGCAAATGCTGGRTTDATYTK } \\ \text { i50F2 } & \text { GATGGAATCCACATTCTCATTAAYATGAAYGG } \\ \text { i50F3 } & \text { GATGGAATCCACATTCTCATNAAYATGAAYGG } \\ \text { i50R3 } & \text { GATGTGACAGCATCCGTGATGAWRTAATCCATRAA } \\ \text { i50R4 } & \text { GGTGATGTGACAGCATCCGTGATGATATAATCCAT } \\ \text { i51F3 } & \text { GATGACGCTATTGTGTTTTGCAATTTYAAYCAGCT } \\ \text { i51F4 } & \text { GATGACGCTATTGTGTTTTGCAAYTTYAAYCAGCT } \\ \text { i51R3 } & \text { ATCAGCCAGTTGTCCTCGACGAACRTGYTCYTCYT } \\ \text { i51R4 } & \text { ATCAGCCAGTTGTCCTCGACGAACATGYTCYTCYT } \\ \text { i52F2 } & \text { GTAACTCATGCTCTCAGAACCACTGARTAYCAYGA } \\ \text { i52R2 } & \text { GCAACAATAAATTGCTTCAATCCHTCVACHGTCA } \\ \text { i53F2 } & \text { ACTGTTCGAGGAGTTATGAGAAGAGGMWTGACDRT } \\ \text { i53R3 } & \text { TTCTTGTTGAACGCCCAAATYTTRTCCCAYTCCAT } \\ \text { i56F2 } & \text { CATCATCTCGGTCAAAACTTCTCCAAVATGTTCRA } \\ \text { i56R3 } & \text { GGCACTCCCTTCAGCTCCCAGTGRTTRWAYTTCCA } \\ \text { i57F2 } & \text { ACAACGTCACCACCACCGAGGATCCVRTNAT } \\ \text { i57R2 } & \text { CTCCGATTTTGTAGGCAACAATATCCCANGARTA }\end{array}$


Table 2: Combinations of EPIC primer pairs used for PCR amplification. The sequences of the primers designed for this study are given in Table 1, the others are provided in Chenuil et al. (2010).

\begin{tabular}{|c|c|c|c|c|c|c|c|c|c|c|c|c|c|}
\hline Primer pair name & $1 \mathrm{a}$ & $1 \mathrm{~b}$ & $1 \mathrm{c}$ & $2 a$ & $3 a$ & $3 \mathrm{~b}$ & $3 c$ & $4 a$ & $5 a$ & $5 b$ & 5c & $5 \mathrm{~d}$ & $5 e$ \\
\hline Forward primer & i1-F & i1-F & i1-F2 & i2-F & i3-F & i3-F2 & i3-F3 & i4-F2 & i5-F & i5-F & i5-F2 & i5-F2 & i5-F2 \\
\hline Reverse primer & $\mathrm{i} 1-\mathrm{R}$ & i1-R2 & i1-R3 & i2-R & i3-R & i3-R2 & i3-R2 & i4-R2 & $\mathrm{i} 5-\mathrm{R}$ & i5-R2 & i5-R3 & i5-R4 & i5-R5 \\
\hline Primer pair name & $8 a$ & $8 \mathrm{~b}$ & $9 a$ & $9 \mathrm{~b}$ & 9c & $11 \mathrm{a}$ & $11 \mathrm{~b}$ & $12 \mathrm{a}$ & $12 \mathrm{~b}$ & $13 a$ & $15 a$ & $15 b$ & $17 \mathrm{a}$ \\
\hline Forward primer & i8-F & i8-F2 & i9-F & i9-F2 & i9-F3 & i11-F & i11-F2 & i12-F & i12-F2 & i13-F2 & i15-F & i15-F2 & i17-F2 \\
\hline Reverse primer & i8- R & i8-R2 & i9-R & i9-R & i9-R2 & i11-R & i11-R2 & i12-R & i12-R2 & i13-R2 & i15-R & i15-R2 & i17-R2 \\
\hline Primer pair name & $19 a$ & $19 \mathrm{~b}$ & 19c & 19d & $21 a$ & $21 \mathrm{~b}$ & $21 \mathrm{c}$ & 21d & $21 \mathrm{e}$ & $21 \mathrm{f}$ & $22 a$ & $22 \mathrm{~b}$ & $22 \mathrm{c}$ \\
\hline Forward primer & i19-F2 & i19-F3 & i19-F & i19-F & i21-F & i21-F & i21-F3 & i21-F3 & i21-F3 & i21-F3 & i22-F & i22-F2 & i22-F2 \\
\hline Reverse primer & i19-R3 & i19-R3 & i19-R & i19-R2 & i21-R & i21-R2 & i21-R & i21-R2 & i21-R3 & i21-R4 & i22-R3 & i22-R2 & i22-R3 \\
\hline Primer pair name & $22 \mathrm{~d}$ & $22 \mathrm{e}$ & $22 f$ & $24 a$ & $24 \mathrm{~b}$ & $25 a$ & $25 b$ & $25 c$ & $26 a$ & $29 a$ & $29 b$ & $29 \mathrm{c}$ & 29d \\
\hline Forward primer & i22-F3 & i22-F3 & i22-F & i24-F2 & i24-F2 & $\mathrm{i} 25-\mathrm{F}$ & i25-F2 & i25-F3 & i26-F3 & i29-F & i29-F & i29-F2 & i29-F3 \\
\hline Reverse primer & i22-R & i22-R3 & i22-R & i24-R2 & i24-R3 & i25-R2 & i25-R2 & i25-R3 & i26-R3 & i29-R & i29-R2 & i29-R2 & i29-R2 \\
\hline Primer pair name & $29 \mathrm{e}$ & $29 \mathrm{f}$ & $30 a$ & $30 \mathrm{~b}$ & $30 \mathrm{c}$ & $30 d$ & $34 a$ & $34 \mathrm{~b}$ & $34 \mathrm{c}$ & $34 d$ & $34 \mathrm{e}$ & $35 a$ & $35 b$ \\
\hline Forward primer & i29-F4 & i29-F4 & i30-F & i30-F & i30-F2 & i30-F3 & i34-F & i34-F & i34-F2 & i34-F2 & i34-F2 & i35-F & i35-F3 \\
\hline Reverse primer & i29-R & i29-R2 & i30-R & i30-R3 & i30-R2 & i30-R2 & i34-R & i34-R4 & i34-R & i34-R3 & i34-R4 & i35-R3 & i35-R3 \\
\hline Primer pair name & $35 c$ & $35 d$ & $35 \mathrm{e}$ & $36 a$ & $36 b$ & $36 c$ & $37 a$ & $37 \mathrm{~b}$ & $38 a$ & $38 \mathrm{~b}$ & $39 a$ & $39 b$ & $40 a$ \\
\hline Forward primer & i35-F3 & i35-F4 & i35-F4 & i36-F & i36-F & i36-F2 & i37-F2 & i37-F3 & i38-F & i38-F2 & i39-F & i39-F2 & i40-F \\
\hline Reverse primer & $\mathrm{i} 35-\mathrm{R} 4$ & i35-R3 & i35-R4 & i36-R & i36-R3 & i36-R3 & i37-R2 & i37-R & i38-R3 & i38-R2 & i39-R & i39-R2 & $\mathrm{i} 40-\mathrm{R} 2$ \\
\hline Primer pair name & $40 \mathrm{~b}$ & $40 c$ & 40d & $40 \mathrm{e}$ & $40 f$ & $41 \mathrm{a}$ & $41 \mathrm{~b}$ & 41c & 41d & $41 \mathrm{e}$ & $42 a$ & $42 \mathrm{~b}$ & $42 \mathrm{c}$ \\
\hline Forward primer & $\mathrm{i} 40-\mathrm{F} 2$ & $\mathrm{i} 40-\mathrm{F} 2$ & i40-F2 & i40-F3 & i40-F3 & $\mathrm{i} 41-\mathrm{F}$ & i41-F2 & i41-F2 & i41-F3 & i41-F3 & i42-F2 & i42-F2 & $\mathrm{i} 42-\mathrm{F}$ \\
\hline Reverse primer & i40-R2 & $\mathrm{i} 40-\mathrm{R} 2$ & i40-R3 & $\mathrm{i} 40-\mathrm{R} 2$ & i40-R3 & i41-R2 & i41-R3 & i41-R4 & i41-R3 & i41-R4 & i42-R3 & i42-R4 & i42-R \\
\hline Primer pair name & $42 d$ & 43a & $43 b$ & $43 c$ & 43d & $44 a$ & $44 \mathrm{~b}$ & $44 c$ & $44 d$ & $44 \mathrm{e}$ & $45 a$ & $45 b$ & $45 c$ \\
\hline Forward primer & $\mathrm{i} 42-\mathrm{F}$ & $\mathrm{i} 43-\mathrm{F}$ & i43-F & i43-F3 & i43-F3 & i44-F2 & i44-F2 & i44-F3 & i44-F3 & i44-F4 & i45-F2 & i45-F2 & i45-F3 \\
\hline Reverse primer & $\mathrm{i} 42-\mathrm{R} 4$ & $\mathrm{i} 43-\mathrm{R}$ & i43-R3 & i43-R3 & i43-R4 & i44-R2 & i44-R3 & $\mathrm{i} 44-\mathrm{R} 2$ & i44-R3 & $\mathrm{i} 44-\mathrm{R} 2$ & $\mathrm{i} 45-\mathrm{R} 2$ & i45-R3 & i45-R2 \\
\hline Primer pair name & $45 \mathrm{~d}$ & $46 a$ & $46 \mathrm{~b}$ & $47 a$ & $47 \mathrm{~b}$ & $48 a$ & $48 \mathrm{~b}$ & $48 \mathrm{c}$ & $48 \mathrm{~d}$ & $48 \mathrm{e}$ & $48 \mathrm{f}$ & $49 a$ & $49 \mathrm{~b}$ \\
\hline Forward primer & i45-F3 & i46-F & i46-F2 & i47-F2 & i47-F2 & i48-F2 & i48-F3 & i48-F4 & i48-F4 & $\mathrm{i} 48-\mathrm{F}$ & $\mathrm{i} 48-\mathrm{F}$ & i49-F & i49-F3 \\
\hline Reverse primer & i45-R3 & $\mathrm{i} 46-\mathrm{R}$ & i46-R2 & i47-R & i47-R2 & i48-R2 & i48-R2 & i48-R & i48-R2 & $\mathrm{i} 48-\mathrm{R}$ & $\mathrm{i} 48-\mathrm{R} 2$ & i49-R4 & i49-R3 \\
\hline Primer pair name & $50 a$ & $50 \mathrm{~b}$ & 50b' & 50c & 50d & $50 \mathrm{e}$ & $50 f$ & $50 \mathrm{~g}$ & 51a & $51 a$ & $51 \mathrm{~b}$ & $51 \mathrm{~b}$ & 51c \\
\hline Forward primer & i50-F & i50-F & i50-F & i50-F2 & i50-F2 & i50-F2 & i50-F3 & i50-F3 & i51-F & i51-F3 & i51-F & i51-F3 & i51-F2 \\
\hline Reverse primer & i50-R & i50-R2 & i50-R2 & i50-R & i50-R3 & i50-R4 & i50-R3 & i50-R4 & i51-R & i51-R2 & i51-R2 & i51-R3 & i51-R \\
\hline Primer pair name & 51c & 51d & 51d & $52 a$ & $52 b$ & $53 a$ & $53 b$ & $53 c$ & $54 a$ & $54 \mathrm{~b}$ & $54 c$ & $54 d$ & $55 a$ \\
\hline Forward primer & i51-F4 & i51-F2 & i51-F4 & i52-F & i52-F2 & i53-F & i53-F & i53-F2 & i54-F & i54-F2 & i54-F & i54-F2 & i55-F \\
\hline Reverse primer & i51-R2 & i51-R2 & i51-R3 & i52-R & i52-R2 & i53-R & i53-R2 & i53-R3 & i54-R & i54-R & i54-R2 & i54-R2 & i55-R \\
\hline Primer pair name & $56 a$ & $56 \mathrm{~b}$ & $57 a$ & $57 \mathrm{~b}$ & $57 \mathrm{c}$ & $58 a$ & & & & & & & \\
\hline Forward primer & i56-F & i56-F2 & i57-F & i57-F & i57-F2 & i58-F & & & & & & & \\
\hline Reverse primer & i56-R & i56-R3 & i57-R & i57-R2 & i57-R2 & i58-R & & & & & & & \\
\hline
\end{tabular}


Table 3 (to be continued): Results per locus for each species.

Letters in the table refer to the primer pairs (see Table 2) for which results were obtained for a given locus and species. Loci which were not tested for a species are in grey cells with the label 'NT'. For other cells, different primer pairs were tested among species according to main plates ( $c f$ materials and methods). The format of the font refers to the amplification pattern obtained: Bold for "Promising", normal for "Introns", italics for “Amplification” (see text for detailed explanations). Background colours indicate the best primer pair result: white for "promising”, yellow for “intron”, blue for “amplification”, black for loci which did not provide any amplicon except, occasionally, primer dimers. When results were obtained from the additional fourth plate, the primer pair letter is underlined. Loci which amplified in none of the species were not reported here (e.g. i24). *: The name of the "main plate” refers to all results in the corresponding rows, except the underlined results, which correspond to plate IV with increased or decreased DNA concentration. 
Table 3 (continued)

\begin{tabular}{|c|c|c|c|c|c|c|c|c|c|c|c|c|c|c|c|}
\hline Main Plate* & Taxon & 1 & 2 & 3 & 4 & 5 & 8 & 9 & 11 & 12 & 15 & 19 & 21 & 22 & 25 \\
\hline CNI-POR-Hedi & Aplysina (1) & b & & & NT & $\mathbf{b} a$ & $\mathrm{a}$ & $\mathrm{a}$ & & $\mathbf{a}$ & $\mathbf{a}$ & NT & ab & & \\
\hline CNI-POR-Hedi & Aurelia (2) & $\mathbf{b}$ & $\mathbf{a}$ & & NT & $\mathbf{b}$ & $\mathrm{a}$ & $\mathrm{b}$ & & $\mathbf{a}$ & $\mathbf{a}$ & NT & & de & \\
\hline CNI-POR-Hedi & Eunicella cav. (3) & $\mathbf{a b}$ & $\mathbf{a}$ & & NT & ba & $\mathrm{a}$ & $\mathrm{b}$ & & $\mathbf{a}$ & $\mathbf{a}$ & NT & ba & ae & \\
\hline CNI-POR-Hedi & Eunicella ver.(2) & ab & $\mathbf{a}$ & & NT & ba & $\mathrm{a}$ & ab & & $\mathbf{a}$ & $\mathbf{a}$ & NT & ba & ae & \\
\hline CNI-POR-Hedi & Hediste (4) & $\mathrm{ab}$ & $\mathbf{a}$ & & NT & ba & $\mathrm{a}$ & $\mathrm{b}$ & & $\mathrm{a}$ & $\mathbf{a}$ & NT & ba & ead & \\
\hline CNI-POR-Hedi & Lophelia (4) & ab & & & NT & $\mathrm{b}$ & & b & & $\mathrm{a}$ & $\mathrm{a}$ & NT & & d & \\
\hline CNI-POR-Hedi & Pelagia (2) & & & & NT & $\mathrm{b}$ & & & & $\mathrm{a}$ & $\mathbf{a}$ & NT & & $\mathbf{a}$ & \\
\hline CNI-POR-Hedi & Rhizostoma (2) & $b$ & $\mathrm{a}$ & & NT & & & & & $\mathbf{a}$ & $\mathrm{a}$ & NT & & & \\
\hline ECDY-Platy & Alvinocaris mar.* (3) & & NT & & $\mathbf{a}$ & C & & c & b & b & & & & & \\
\hline ECDY-Platy & Alvinocaris mur.* (3) & & NT & & $\mathbf{a}$ & c & b & C & $\mathrm{b}$ & $b$ & & & & & \\
\hline ECDY-Platy & Hemimysis (3) & & NT & & & & & & & & & & $\underline{\mathbf{b}}$ & & \\
\hline ECDY-Platy & Mesopodopsis (4) & & NT & & & & & & b & & & & & & \\
\hline ECDY-Platy & Litoditis (2) & & NT & & & & & & & $b$ & & & & & \\
\hline ECDY-Platy & Platynereis (2) & & NT & c & $\bar{a}$ & c & $\mathbf{b}$ & C & $\mathrm{b}$ & b & & & & & \\
\hline ECDY-Platy & Rimicaris (4) & & NT & & $\mathbf{a}$ & & & C & & b & & & & & \\
\hline LOPHO-ECHI & Acanthaster (2) & ba & $a$ & $\mathrm{a}$ & NT & $\mathrm{ab}$ & $\mathrm{a}$ & $\mathbf{b} a$ & $\mathbf{a}$ & & & $C$ & & afe & \\
\hline LOPHO-ECHI & Crepidula (4) & $\mathrm{a}$ & & & NT & $\mathbf{b}$ & $\mathrm{a}$ & $\mathrm{b}$ & & & & & & & \\
\hline LOPHO-ECHI & Eunicidae (3) & ab & $\mathbf{a}$ & & NT & b & & b & $\mathbf{a}$ & & & 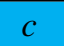 & ab & afe & b \\
\hline LOPHO-ECHI & Ophiocten (3) & $\mathbf{a b}$ & & & NT & $\mathbf{b}$ & $\mathrm{a}$ & $\mathrm{b}$ & & & & c & & $\mathrm{a}$ & \\
\hline LOPHO-ECHI & Ophioderma (3) & $a b$ & $\mathrm{a}$ & & NT & ab & $\mathrm{a}$ & b & $\mathrm{a}$ & & & & ab & afe & \\
\hline LOPHO-ECHI & Ostrea (3) & $\mathrm{ab}$ & $\mathrm{a}$ & $\mathbf{a}$ & NT & ab & $\mathbf{a}$ & b & $\mathrm{a}$ & & & & ba & aef & $\mathbf{b}$ \\
\hline LOPHO-ECHI & Platynereis (2) & & $\mathbf{a}$ & & NT & ab & $\mathrm{a}$ & $\mathrm{b}$ & & & & & $\mathbf{a b}$ & afe & $\mathbf{b}$ \\
\hline IV (i21-i51) & Mya (4) & NT & NT & NT & NT & NT & NT & NT & NT & NT & NT & NT & $\underline{\mathbf{b}}$ & NT & NT \\
\hline
\end{tabular}


Table 3 (continued)

\begin{tabular}{|c|c|c|c|c|c|c|c|c|c|c|c|c|c|c|c|}
\hline Main Plate* & Taxon & 26 & 29 & 30 & 34 & 35 & 36 & 37 & 38 & 39 & 40 & 41 & 42 & 43 & 44 \\
\hline CNI-POR-Hedi & Aplysina (1) & NT & & & $\mathbf{a}$ & & & & & $\mathrm{a}$ & b & & & & $e$ \\
\hline CNI-POR-Hedi & Aurelia (2) & NT & $\mathbf{b}$ & $\mathrm{ab}$ & & & & b & & $\mathrm{a}$ & & & & $\mathrm{a}$ & e \\
\hline CNI-POR-Hedi & Eunicella cav. (3) & NT & abef & ba & abc & $\mathbf{a}$ & & $\mathrm{b}$ & & $a$ & b & & d & $\mathbf{a} b$ & e \\
\hline CNI-POR-Hedi & Hediste (4) & NT & abef & $\mathbf{b}$ & abc & $\mathbf{a}$ & & & & $\mathbf{a}$ & & & d & $\mathbf{a}$ & $\mathbf{e}$ \\
\hline CNI-POR-Hedi & Lophelia (4) & NT & $\mathrm{b}$ & $\mathrm{b}$ & $\mathbf{a}$ & & & & & & $\mathrm{b}$ & & & & e \\
\hline CNI-POR-Hedi & Pelagia (2) & NT & & b & & & & $\mathrm{b}$ & & $\mathrm{a}$ & & & & & \\
\hline ECDY-Platy & Alvinocaris mar. (3) & $\mathrm{a}$ & cd & & & ed & C & $\mathrm{a}$ & $\mathbf{b}$ & $\mathrm{b}$ & dce & $\overline{\mathbf{b c}}$ & & & $\mathrm{a}$ \\
\hline ECDY-Platy & Alvinocaris mur. (3) & $\mathrm{a}$ & cd & & & ecd & c & a & b & $\mathrm{b}$ & dfce & b & & & $\mathrm{a}$ \\
\hline ECDY-Platy & Hemimysis (3) & & C & & & & & & & & C & & & & \\
\hline ECDY-Platy & Mesopodopsis (4) & $\mathbf{a}$ & & & & $\mathrm{d}$ & & & & & & & & & $a$ \\
\hline ECDY-Platy & Litoditis (2) & & c & & & dec & $c$ & & & & & & $\mathbf{a}$ & & d \\
\hline ECDY-Platy & Platynereis (2) & $a$ & cd & & & de & c & $\mathrm{a}$ & b & $\mathrm{b}$ & fcde & ebc & $\mathbf{a}$ & & ad \\
\hline ECDY-Platy & Rimicaris (4) & $\mathbf{a}$ & cd & & & c & c & $\mathbf{a}$ & $\mathrm{b}$ & $b$ & $c d$ & bc & $\mathbf{a}$ & & \\
\hline LOPHO-ECHI & Acanthaster (2) & NT & bae & $\mathrm{a}$ & $\mathbf{a}$ & $\mathbf{a}$ & $\mathbf{a b}$ & b & & $\mathbf{a}$ & b & & NT & $\mathbf{a}$ & $\mathbf{e}$ \\
\hline LOPHO-ECHI & Eunicidae (3) & NT & be & ab & & $\mathbf{a}$ & $\mathbf{a b}$ & b & & & b & & NT & & \\
\hline LOPHO-ECHI & Ophiocten (3) & NT & & & & & $\mathrm{b}$ & & & $\mathbf{a}$ & $b$ & & NT & $\mathrm{ab}$ & $e$ \\
\hline LOPHO-ECHI & Ophioderma (3) & NT & bea & $\mathrm{b}$ & & $\mathrm{a}$ & $\mathbf{a b}$ & $\mathrm{b}$ & & $\mathbf{a}$ & b & & NT & $\mathbf{a}$ & $e$ \\
\hline LOPHO-ECHI & Ostrea (3) & NT & afbe & $\mathbf{a b}$ & abce & $\mathbf{a}$ & $\mathrm{ab}$ & $\mathrm{b}$ & $\mathbf{a}$ & $a$ & b & & NT & $\mathrm{ab}$ & $e$ \\
\hline LOPHO-ECHI & Platynereis (2) & NT & $a b$ & & & & b & b & & $\mathbf{a}$ & & & NT & & $e$ \\
\hline IV (i21-i51) & Mya (4) & NT & NT & NT & NT & NT & NT & NT & NT & NT & NT & NT & NT & NT & NT \\
\hline
\end{tabular}


Table 3 (end)

\begin{tabular}{|c|c|c|c|c|c|c|c|c|c|c|c|c|c|c|c|}
\hline Main Plate* & Taxon & 45 & 46 & 47 & 48 & 49 & 50 & 51 & 52 & 53 & 54 & 55 & 56 & 57 & 58 \\
\hline CNI-POR-Hedi & Aplysina (1) & NT & $\mathbf{a}$ & & & & $a b$ & $b$ & & $a$ & NT & & $\mathbf{a}$ & & \\
\hline CNI-POR-Hedi & Aurelia (2) & NT & $\mathbf{a}$ & & & & ac & $\mathrm{bdc}$ & & $\mathrm{a}$ & NT & & & $\mathbf{a}$ & \\
\hline CNI-POR-Hedi & Eunicella cav. (3) & NT & $\mathbf{a}$ & & cd & & ca & $a b c d$ & & ab & NT & & $\mathbf{a}$ & & $\mathbf{a}$ \\
\hline CNI-POR-Hedi & Eunicella ver.(2) & NT & $\mathbf{a}$ & & d & & ca & abcd & & $\mathbf{a b}$ & NT & & $\mathbf{a}$ & b & $\mathbf{a}$ \\
\hline CNI-POR-Hedi & Hediste (4) & NT & $\mathrm{a}$ & & c & & abc & $\mathrm{b}$ & & ab & NT & & $\mathbf{a}$ & & $\mathbf{a}$ \\
\hline CNI-POR-Hedi & Lophelia (4) & NT & & & & & & $b$ & & $\mathbf{b}$ & NT & & & & \\
\hline CNI-POR-Hedi & Pelagia (2) & NT & & & & & & $a b c$ & & a & NT & & & & \\
\hline CNI-POR-Hedi & Rhizostoma (2) & NT & & & & & & $b$ & & & NT & & & & $\mathbf{a}$ \\
\hline ECDY-Platy & Alvinocaris mar. (3) & $a b c d$ & & & & & cef & fe & & & NT & NT & & & NT \\
\hline ECDY-Platy & Alvinocaris mur. (3) & $a b c d$ & & & & & ef & fe & & & NT & NT & & & NT \\
\hline ECDY-Platy & Hemimysis(3) & $a b c d$ & & & & & & & & & NT & NT & & & NT \\
\hline ECDY-Platy & Mesopodopsis (4) & $a$ & & & & & & & & & NT & NT & & & NT \\
\hline ECDY-Platy & Litoditis (2) & & & & & & cend & $g$ & & & NT & NT & & & NT \\
\hline ECDY-Platy & Platynereis (2) & $a b c d$ & & & & $9 b$ & ef & egf & & & NT & NT & & & NT \\
\hline ECDY-Platy & Rimicaris (4) & $a b c d$ & & & $\mathbf{b}$ & & $\underline{\text { cd }}$ & fe & & & NT & NT & & & NT \\
\hline LOPHO-ECHI & Acanthaster (2) & NT & & & ecd & & $\overline{\mathbf{a b}}$ & acd & $a$ & $a b$ & ac & & & $\mathbf{a b}$ & \\
\hline LOPHO-ECHI & Crepidula (4) & NT & & & & & $\underline{\mathrm{b}} a$ & $b c d$ & & $b$ & & & & & $a$ \\
\hline LOPHO-ECHI & Eunicidae (3) & NT & $\mathrm{a}$ & $b$ & $\mathrm{f}$ & $9 a$ & $\mathrm{a}$ & bcd & & $\mathrm{b} a$ & bca & & & $\mathbf{a b}$ & \\
\hline LOPHO-ECHI & Ophiocten (3) & NT & a & & & & abc & abcd & & $b$ & & & & & \\
\hline LOPHO-ECHI & Ophioderma (3) & NT & $\mathbf{a}$ & $\mathrm{b}$ & & & & acdb & $a$ & $\mathrm{~b}$ & abc & & & & \\
\hline LOPHO-ECHI & Ostrea (3) & NT & a & b & def & $9 a$ & bb'c & & $a$ & $a b$ & acdb & a & & ab & $\mathrm{a}$ \\
\hline LOPHO-ECHI & Platynereis (2) & NT & $\mathbf{a}$ & & $\mathbf{e}$ & & ab'c & abcd & & $b$ & dc & $a$ & & & \\
\hline IV (i21-i51) & Myа (4) & NT & NT & NT & NT & NT & $\underline{\mathbf{a b}}$ & NT & NT & NT & NT & NT & NT & NT & NT \\
\hline
\end{tabular}

*: These Alvinocaris species were later shown to belong to a single genetic entity (with the mitochondrial COI and $18 \mathrm{~S}$ rDNA genes), the few differences being due to individual variation (Teixeira et al, in press). 
Table 4: Summary of results per species ranked according to the plate (note that Platynereis was studied on two distinct plates): numbers correspond to all loci obtaining P, I or A results, respectively. When several primer pairs were tested for a locus we only considered the best of the results $(\mathrm{P}>\mathrm{I}>\mathrm{A})$ to characterize the locus. The proportion of amplicons which are too short to contain an intron is the number of " $\mathrm{A}$ ” divided by “A+I+P”. Extreme values commented in the discussion are highlighted in bold except for Hemimysis. The 50\% value for Hemimysis is not considered as reliable since its estimation is probably uncertain due to overall low positive results (see text).

\begin{tabular}{|c|c|c|c|c|c|}
\hline $\begin{array}{l}\text { Taxa (number of } \\
\text { individuals) }\end{array}$ & $\mathrm{P}$ & I & A & $(\mathrm{A}+\mathrm{I}+\mathrm{P})$ & $\begin{array}{l}\text { Amplicons too short to } \\
\text { contain an intron }\end{array}$ \\
\hline \multicolumn{6}{|l|}{ CNI-POR-Hedi plate } \\
\hline Aplysina (1) & 10 & 3 & 3 & 16 & $19 \%$ \\
\hline Aurelia (2) & 12 & 7 & 0 & 19 & 0 \% \\
\hline Eunicella cav. (3) & 18 & 6 & 2 & 26 & $8 \%$ \\
\hline Eunicella ver.(2) & 19 & 5 & 2 & 26 & $8 \%$ \\
\hline Hediste (4) & 17 & 7 & 0 & 24 & $0 \%$ \\
\hline Lophelia (4) & 6 & 6 & 1 & 13 & $8 \%$ \\
\hline Pelagia (2) & 3 & 5 & 1 & 9 & $11 \%$ \\
\hline Rhizostoma (2) & 6 & 2 & 3 & 11 & $27 \%$ \\
\hline \multicolumn{6}{|l|}{ ECDY-Platy plate } \\
\hline Alvinocaris mar. (3) & 9 & 8 & 3 & 20 & $15 \%$ \\
\hline Alvinocaris mur. (3) & 9 & 7 & 3 & 19 & $16 \%$ \\
\hline Hemimysis(3) & 1 & 1 & 2 & 4 & $50 \%$ \\
\hline Mesopodopsis (4) & 2 & 1 & 2 & 5 & $40 \%$ \\
\hline Litoditis (2) & 4 & 1 & 3 & 8 & $38 \%$ \\
\hline Platynereis (2) & 12 & 4 & 6 & 22 & $27 \%$ \\
\hline Rimicaris (4) & 12 & 1 & 4 & 17 & $24 \%$ \\
\hline \multicolumn{6}{|l|}{ LOPHO-ECHI plate } \\
\hline Acanthaster (2) & 19 & 4 & 4 & 27 & $15 \%$ \\
\hline Crepidula (4) & 1 & 6 & 6 & 13 & $46 \%$ \\
\hline Eunicidae (3) & 13 & 7 & 3 & 23 & $13 \%$ \\
\hline Ophiocten (3) & 6 & 6 & 3 & 15 & $20 \%$ \\
\hline Ophioderma (3) & 10 & 11 & 2 & 23 & $9 \%$ \\
\hline Ostrea (3) & 21 & 7 & 2 & 30 & $7 \%$ \\
\hline Platynereis (2) & 13 & 2 & 4 & 19 & $21 \%$ \\
\hline
\end{tabular}


Table 5: Expected consequences of some molecular and evolutionary processes on the patterns observed.

\begin{tabular}{|c|c|c|c|}
\hline & $\begin{array}{c}\text { Total amplification } \\
\text { success } \\
\mathrm{A}+\mathrm{P}+\mathrm{I} \\
\end{array}$ & $\begin{array}{c}\text { Proportion of amplicons too } \\
\text { short to contain an intron: } \\
\mathrm{A} /(\mathrm{A}+\mathrm{P}+\mathrm{I}) \\
\end{array}$ & Taxon \\
\hline Damaged DNA & $y$ & $\begin{array}{c}\boldsymbol{\lambda} \text { (small target DNA less } \\
\text { damaged) }\end{array}$ & $\begin{array}{l}\text { Hemimysis } \\
\text { Mesopodopsis } \\
\text { (Litoditis) }\end{array}$ \\
\hline $\begin{array}{l}\text { r-strategy : selection for } \\
\text { rapid replication rate, thus } \\
\text { for smaller introns }\end{array}$ & $\begin{array}{c}\text { No effect } \\
\text { (P or I loci are turned into } \\
\text { A) }\end{array}$ & $\pi$ & Crepidula \\
\hline $\begin{array}{l}\text { High genome } \\
\text { evolutionary rate }\end{array}$ & ע (more mispriming) & No effect & $\begin{array}{l}\text { Hemimysis* } \\
\text { Mesopodopsis* } \\
\text { (Litoditis*) }\end{array}$ \\
\hline
\end{tabular}

*: These ecdysozoan species display an increased proportion of short amplicons, but the estimation of this proportion is affected by a high variance, due to their low number of successful amplifications $(\mathrm{A}+\mathrm{P}+\mathrm{I})$; thus we do not rule out the possibility of a role of high evolutionary rate (having in theory no effect on this proportion. 